\title{
REMARK ON THE MODULUS OF COMPACT OPERATORS
}

\author{
BY ULRICH KRENGEL ${ }^{1}$
}

Communicated by R. C. Buck, September 1, 1965

If $X$ and $Y$ are Banach lattices (see Day [1]) the linear continuous operators $T$ from $X$ to $Y$ are partially ordered by: $T_{1} \geqq T_{2}$ if and only if $T_{1} f \geqq T_{2} f$ for all $0 \leqq f \in X$. For some kinds of pairs $(X, Y)$, e.g. $X=Y=L_{1}$ or $L_{\infty}$, the continuous operators have been shown to form a Banach lattice (see Kantorovitch [2]). This note contains a surprising example, showing that the modulus of a compact operator need not necessarily be compact, and a sufficient condition under which the modulus will be compact.

EXAMPLE. We shall modify an example in [3]: Let $\Omega$ be the union of disjoint sets $\Omega_{n}(n=1,2, \cdots)$ where $\Omega_{n}$ consists of $2^{n}$ points $x_{n i}\left(i=1, \cdots, 2^{n}\right)$, with measure 1 each. Define an infinite matrix $A=\left(a_{i k}\right)$ by induction:

$$
A_{1}=\left(\begin{array}{ll}
+1 & +1 \\
+1 & -1
\end{array}\right), \quad A_{j+1}=\left(\begin{array}{ll}
+A_{j} & +A_{j} \\
+A_{j} & -A_{j}
\end{array}\right)
$$

where $A_{n}$ is the matrix of the first $2^{n}$ rows and columns in $A$. If $\chi_{\{x\}}$ is the characteristic function of $\{x\}$ we define the operator $S_{n}$ in $L_{2}(\Omega)$ by:

$$
\chi_{\left\{x_{n k}\right\}} S_{n}=2^{-n} \sum_{i=1}^{2 n} a_{i k} \chi\left\{x_{n i}\right) \quad \text { and } \quad \chi_{\left\{x_{m k}\right\}} S_{n}=0 \text { for } m \neq n .
$$

$\left|S_{n}\right|$ is obtained by using $\left|a_{i k}\right|=1$ instead of $a_{i k}$ in (1). In [3, p. 171] the operators $T_{n}=2^{n / 2} S_{n}$ were investigated and the norms observed to be $\left\|T_{n}\right\|=1,\left\|\left|T_{n}\right|\right\|=2^{n / 2} . S=\sum_{n=1}^{\infty} S_{n}$ is a continuous operator with $|S|=\sum_{n=1}^{\infty}\left|S_{n}\right|$. Since for each $N, \sum_{n=1}^{N} S_{n}$ is a compact operator and these tend to $S$ in norm, $S$ is compact. To see that $|S|$ is not compact look at the functions $f_{n}$ which are $=2^{-n / 2}$ on $\Omega_{n}$ and 0 elsewhere. They satisfy $f_{n}=|S| f_{n}$ and $\left\|f_{n}\right\|=1$.

If the modulus $|T|$ of an operator $T$ exists, it has the form

$$
|T| f=\sup _{|\theta| \geqq f}|T g| \quad \text { for } f \in X^{+}=\{h \in X: h \geqq 0\}
$$

(see [3]). ${ }^{2}$ In [3] it has been shown, that the modulus of any compact

1 This work was prepared with partial support of the National Science Foundation GP-2593.

${ }^{2}$ In [3] the definition of a Banach lattice unnecessarily is slightly more special than in [1]. 
operator $T: X \rightarrow Y$ is compact, if $X$ is an $L$-space (see Day [1]) and the monotone convergence theorem holds in $Y$.

Theorem. If $X$ is any Banach lattice and $Y$ is an $M$-space (see [1]) the compact operators $T$ from $X$ to $Y$ form a Banach lattice.

The proof is based on the following lemma:

Lemma. If $Y$ is a $M$-space and $C \subseteq Y$ is conditionally compact, $\sup \{f: f \in A\}$ exists for all $A \subseteq C$ and the set of all such suprema is conditionally compact.

The lemma is proved by representing $Y$ as a subspace of the space $C(\Omega)$ of continuous functions on some compact space $\Omega$ and by an application of the Arzela-Ascoli theorem.

\section{REFERENCES}

1. M. Day, Normed linear spaces, Springer, Berlin, 1958.

2. L. Kantorovitch, Linear operations in semi-ordered spaces, Mat. Sb. (N.S.) 49 (1940), 209-284.

3. U. Krengel, Ueber den Absolutbetrag stetiger linearer Operatoren und seine Anwendung auf ergodische Zerlegungen, Math. Scand. 13 (1963), 151-187.

University of CaLifornia, Berkeley 